\title{
1S-2S energy shift in muonic hydrogen
}

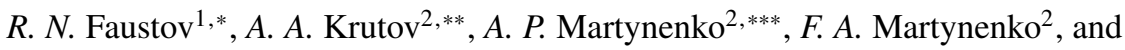 \\ O. S. Sukhorukova ${ }^{2}$ \\ ${ }^{1}$ FRC Computer Science and Control RAS, 119991, Moscow, Russia \\ ${ }^{2}$ Samara National Research University named after S. P. Korolev, 443086, Samara, Russia
}

\begin{abstract}
We calculate corrections of orders $\alpha^{4}, \alpha^{5}, \alpha^{6}$ to the $(1 S-2 S)$ fine structure interval in muonic hydrogen $(\mu p)$, muonic tritium $(\mu t)$ and muonic helium ion $\left(\left(\mu_{2}^{3} \mathrm{He}\right)^{+}\right)$. They are determined by the effects of vacuum polarization, nuclear structure and recoil and relativistic corrections. The nuclear structure effects are taken into account in terms of the charge radii of the nuclei in onephoton interaction and in terms of electromagnetic form-factors in the case of two-photon interaction. The obtained results for the $(1 S-2 S)$ splitting can be used for a comparison with future experimental data.
\end{abstract}

\section{Introduction}

The current experimental program to study the fine and hyperfine structure of the simplest muonic atoms is successfully implemented, starting with 2010 , when transitions $\left(2 S_{1 / 2}^{F=1}-\right.$ $\left.2 P_{3 / 2}^{F=2}\right)$ and $\left(2 S_{1 / 2}^{F=0}-2 P_{3 / 2}^{F=1}\right)$ were measured in muonic hydrogen [1]. The experiments with muonic hydrogen have shown that there is a significant discrepancy between the values of charge radii of the proton and deuteron obtained from experiments with electronic and muonic atoms [2-4]. One of the possible tasks of future experiments may be measuring the $(2 S-$ $1 S$ ) transition frequency in the muonic hydrogen and muonic helium ions. It requires an appropriate precise theoretical study. In the case of a hydrogen atom the interval of a large fine structure $(1 S-2 S)$ was measured with very high accuracy. Despite the appearance of numerous papers $[5-17,21]$ devoted to investigations of different corrections in the energy spectrum of light muonic atoms the value of energy interval $(1 S-2 S)$ has not been studied with good accuracy. So, the goal of this work is to calculate the fine structure energy interval $(1 S-2 S)$ in a number of muonic atoms included in CREMA experiments.

\section{Basic vacuum polarization, nuclear structure and relativistic corrections}

Our approach to the calculation of corrections in the $(1 S-2 S)$ shift is based on the quasipotential method in quantum electrodynamics $[6,7,13]$. The two-particle bound state is described by the Schrödinger equation. The leading order contribution to the particle interaction operator is determined by the Breit Hamiltonian. The fine structure of the energy levels

\footnotetext{
*e-mail: faustov@ccas.ru

**e-mail: aakrutov@ rambler.ru

***e-mail: a.p.martynenko@samsu.ru
} 
of $S$-states of hydrogen-like atoms consisting of the particles with masses $m_{1}, m_{2}$ can be written with the accuracy $O\left((Z \alpha)^{6}\right)$ ( $\mu$ is the reduced mass) [5]:

$$
E_{n}=m_{1}+m_{2}-\frac{\mu^{2}(Z \alpha)^{2}}{2 n^{2}}-\frac{\mu(Z \alpha)^{4}}{2 n^{3}}\left[1-\frac{3}{4 n}+\frac{\mu^{2}}{4 m_{1} m_{2} n}\right]-\frac{m_{1}(Z \alpha)^{6}}{16 n^{6}}\left(2 n^{3}+6 n^{2}-12 n+5\right) .
$$

Taking into account the modification of the Coulomb potential due to the vacuum polarization (VP) effect in the coordinate representation

$$
V_{v p}^{C}(r)=\frac{\alpha}{3 \pi} \int_{1}^{\infty} d \xi \rho(\xi)\left(-\frac{Z \alpha}{r} e^{-2 m_{e} \xi r}\right), \quad \rho(\xi)=\frac{\sqrt{\xi^{2}-1}\left(2 \xi^{2}+1\right)}{\xi^{4}},
$$

we can present the contribution of one-loop electron VP to the shift of $1 \mathrm{~S}$ and $2 \mathrm{~S}$ energy levels in the form:

$$
\begin{gathered}
\Delta E_{v p}(1 S)=-\frac{4 \mu(Z \alpha)^{2} \alpha}{3 \pi} \int_{1}^{\infty} \rho(\xi) d \xi \int_{0}^{\infty} x d x e^{-2 x\left(1+\frac{m_{e} \xi}{W}\right)} \\
\Delta E_{v p}(2 S)=-\frac{\mu(Z \alpha)^{2} \alpha}{6 \pi} \int_{1}^{\infty} \rho(\xi) d \xi \int_{0}^{\infty} x d x\left(1-\frac{x}{2}\right)^{2} e^{-x\left(1+\frac{2 m_{e} \xi}{W}\right)} .
\end{gathered}
$$

The corresponding numerical correction in the interval $(2 S-1 S)$ is presented in Tab. 1. In the same way we calculate two- and three-loop VP corrections in $1 \gamma$ interaction (see Fig. 1).

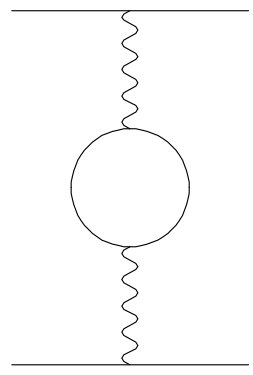

a

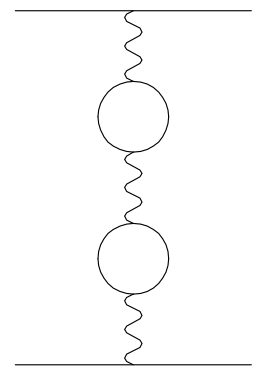

$\mathrm{b}$

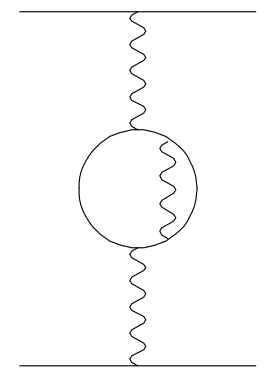

$\mathrm{c}$

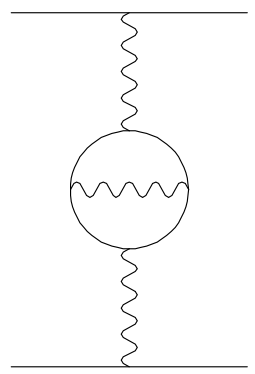

d

Figure 1. The effects of one-loop, two-loop vacuum polarization in one-photon interaction

In the second order of perturbation theory (SOPT) the energy correction is determined by the reduced Coulomb Green's functions $\tilde{G}_{1 S}, \tilde{G}_{2 S}$. They were obtained in a convenient compact form in [18]. In the case of Green's functions $\tilde{G}_{1 S}, \tilde{G}_{2 S}$ with one zero argument we have:

$$
\begin{gathered}
\tilde{G}_{1 S}(\mathbf{r}, 0)=\frac{Z \alpha \mu^{2}}{4 \pi} \frac{e^{-x}}{x} g_{1 S}(x), \quad g_{1 S}(x)=4 x(\ln 2 x+C)+4 x^{2}-10 x-2, \quad x=\mu Z \alpha r, \\
\tilde{G}(2 S)(\mathbf{r}, 0)=-\frac{Z \alpha \mu^{2}}{4 \pi} \frac{e^{-x / 2}}{2 x} g_{2 S}(x), \quad g_{2 S}(x)=4 x(x-2)(\ln x+C)+x^{3}-13 x^{2}+6 x+4 .
\end{gathered}
$$

In the second order of perturbation theory we have a number of contributions from vacuum polarization on orders of $\alpha^{2}(Z \alpha)^{2}$ and $\alpha(Z \alpha)^{4}$ to the shift $(1 S-2 S)$, which are shown in the diagrams in Fig. 2. 

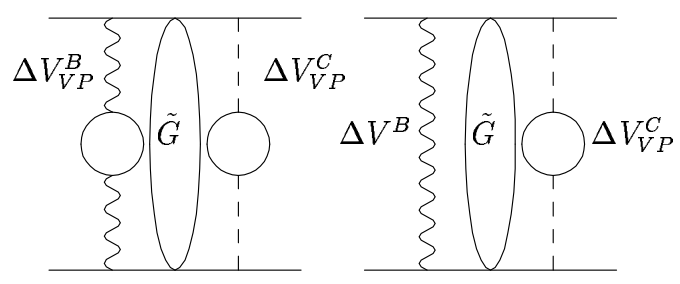

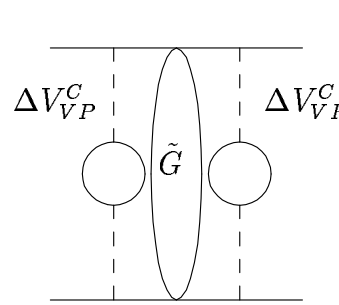

c

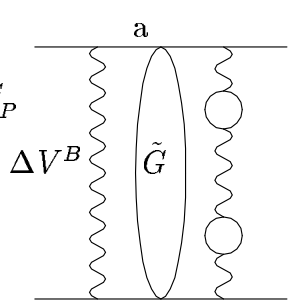

d

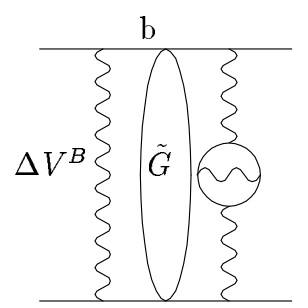

$\mathrm{e}$

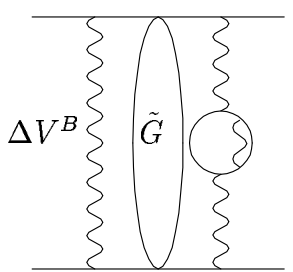

f

Figure 2. Effects of one-loop and two-loop VP in the second order perturbation theory. $\tilde{G}$ is the reduced Coulomb Green's function

The muon wave function of muonic atom is strongly overlapped with the nuclear charge distribution region. In the leading order $\alpha^{4}$, the effect of the nuclear structure can be expressed in terms of the nuclear charge radius $r_{N}$ [5]:

$$
\Delta E_{s t r}(2 S-1 S)=-\frac{7 \mu^{3}(\mathrm{Z} \alpha)^{4}}{12}<r_{N}^{2}>= \begin{cases}-36.382164 r_{p}^{2} \mathrm{meV}, & (\mu p), \\ -44.854681 r_{t}^{2} \mathrm{meV}, & (\mu t), \\ -717.660184 r_{h e}^{2} \mathrm{meV}, & \left(\left(\mu^{3} \mathrm{He}\right)^{+}\right) .\end{cases}
$$

Nuclear corrections in two-photon interaction of order $(Z \alpha)^{5}$ are determined by the integral expression:

$$
\begin{array}{r}
\Delta E_{s t r}^{2 \gamma}(n S)=-\frac{\mu^{3}(Z \alpha)^{5}}{\pi n^{3}} \delta_{l 0} \int_{0}^{\infty} \frac{d k}{k} V(k), \quad V(k)=\frac{2\left(F_{1}^{2}-1\right)}{m_{1} m_{2}}+\frac{8 m_{1}\left[F_{2}(0)+4 m_{2}^{2} F_{1}^{\prime}(0)\right]}{m_{2}\left(m_{1}+m_{2}\right) k} \\
+\frac{k^{2}}{2 m_{1}^{3} m_{2}^{3}}\left[2\left(F_{1}^{2}-1\right)\left(m_{1}^{2}+m_{2}^{2}\right)+4 F_{1} F_{2} m_{1}^{2}+3 F_{2}^{2} m_{1}^{2}+\frac{\sqrt{k^{2}+4 m_{1}^{2}}}{2 m_{1}^{3} m_{2}\left(m_{1}^{2}-m_{2}^{2}\right) k} \times\right. \\
\quad\left\{k^{2}\left[2\left(F_{1}^{2}-1\right) m_{2}^{2}+4 F_{1} F_{2} m_{1}^{2}+3 F_{2}^{2} m_{1}^{2}\right]-8 m_{1}^{4} F_{1} F_{2}+\frac{16 m_{1}^{4} m_{2}^{2}\left(F_{1}^{2}-1\right)}{k^{2}}\right\}- \\
-\frac{\sqrt{k^{2}+4 m_{2}^{2}} m_{1}}{2 m_{2}^{3}\left(m_{1}^{2}-m_{2}^{2}\right) k}\left\{k^{2}\left[2\left(F_{1}^{2}-1\right)+4 F_{1} F_{2}+3 F_{2}^{2}\right]-8 m_{2}^{2} F_{1} F_{2}+\frac{16 m_{2}^{4}\left(F_{1}^{2}-1\right)}{k^{2}}\right\}
\end{array}
$$

where $F_{1}, F_{2}$ are the Dirac and Pauli nucleus electromagnetic form factors. The recoil correction of the fifth order in $(Z \alpha)$ is also connected with two-photon exchange amplitudes, in which the nucleus is treated as a point particle [5]:

$$
\Delta E_{\text {rec }}^{(Z \alpha)^{5}}=\frac{\mu^{3}(Z \alpha)^{5}}{m_{1} m_{2} \pi n^{3}}\left[\frac{2}{3} \delta_{l 0} \ln \frac{1}{Z \alpha}-\frac{8}{3} \ln k_{0}(n, l)-\frac{1}{9} \delta_{l 0}-\frac{7}{3} a_{n}-\frac{2}{m_{2}^{2}-m_{1}^{2}} \delta_{l 0}\left(m_{2}^{2} \ln \frac{m_{1}}{\mu}-m_{1}^{2} \ln \frac{m_{2}}{\mu}\right)\right] .
$$


The amplitudes of interactions between particles, which contain the effects of both - the nuclear structure and vacuum polarization (see Fig. 3), should be also considered in obtaining the total value of the Lamb shift. The operator of interaction between particles corresponding to the diagram in Fig. 3(b) has the form:

$$
\Delta V_{s t r}^{v p}(r)=\frac{2}{3} \pi Z \alpha<r_{N}^{2}>\frac{\alpha}{3 \pi} \int_{1}^{\infty} \rho(\xi) d \xi\left[\delta(\mathbf{r})-\frac{m_{e}^{2} \xi^{2}}{\pi r} e^{-2 m_{e} \xi r}\right] .
$$

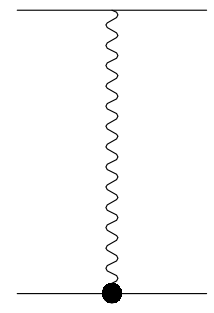

a

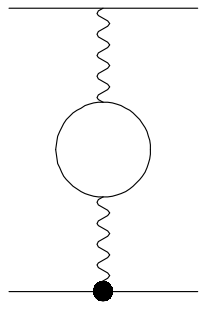

b

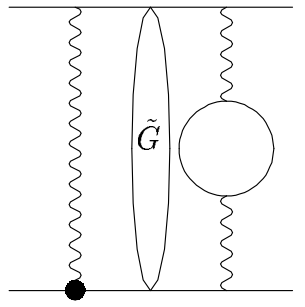

C

Figure 3. Nuclear structure and one-loop vacuum polarization corrections in first order PT (FOPT) and second order PT (SOPT)

The effects of VP in the Breit Hamiltonian were investigated in $[7,8,19]$. The corrections from one-loop VP have the following form:

$$
\begin{gathered}
\Delta V_{v p}^{B}(r)=\frac{\alpha}{3 \pi} \int_{1}^{\infty} \rho(\xi) d \xi \sum_{i=1}^{4} \Delta V_{i, v p}^{B}(r), \\
\Delta V_{1, v p}^{B}=\frac{Z \alpha}{8}\left(\frac{1}{m_{1}^{2}}+\frac{\delta_{I}}{m_{2}^{2}}\right)\left[4 \pi \delta(\mathbf{r})-\frac{4 m_{e}^{2} \xi^{2}}{r} e^{-2 m_{e} \xi r}\right], \\
\Delta V_{2, v p}^{B}=-\frac{Z \alpha m_{e}^{2} \xi^{2}}{m_{1} m_{2} r} e^{-2 m_{e} \xi r}\left(1-m_{e} \xi r\right), \\
\Delta V_{3, v p}^{B}=-\frac{Z \alpha}{2 m_{1} m_{2}} p_{i} \frac{e^{-2 m_{e} \xi r}}{r}\left[\delta_{i j}+\frac{r_{i} r_{j}}{r^{2}}\left(1+2 m_{e} \xi r\right)\right] p_{j}, \\
\Delta V_{4, v p}^{B}=\frac{Z \alpha}{r^{3}}\left(\frac{1}{4 m_{1}^{2}}+\frac{1}{2 m_{1} m_{2}}\right) e^{-2 m_{e} \xi r}\left(1+2 m_{e} \xi r\right)\left(\mathbf{L} \sigma_{1}\right) .
\end{gathered}
$$

These terms give the corrections of order $\alpha(Z \alpha)^{4}$ in the energy spectrum. The corrections of order $\alpha^{2}(Z \alpha)^{4}$ appear from two-loop VP corrections to the Breit Hamiltonian. Total relativistic contributions are presented in Tab. 1.

In the sixth order in $\alpha$, a correction to the nuclear structure with a vacuum polarization effect also exists and is described by the two-photon exchange amplitudes in Fig. 4. The corresponding particle interaction operator is obtained in the integral form:

$$
\Delta E_{s t r, v p}^{2 \gamma}(n S)=-\frac{2 \mu^{3} \alpha(Z \alpha)^{5}}{\pi^{2} n^{3}} \int_{0}^{\infty} k V(k) d k \int_{0}^{1} \frac{v^{2}\left(1-\frac{v^{2}}{3}\right) d v}{k^{2}\left(1-v^{2}\right)+4 m_{e}^{2}} .
$$


Table 1. Corrections in the $(2 S-1 S)$ fine structure interval in muonic atoms $\mu p, \mu t,\left(\mu_{2}^{3} H e\right)^{+}$

\begin{tabular}{|c|c|c|c|}
\hline Contribution to the $(2 S-1 S)$ shift & $\mu p, \mathrm{meV}$ & $\mu t, \mathrm{meV}$ & $\left(\mu_{2}^{3} \mathrm{He}\right)^{+}, \mathrm{meV}$ \\
\hline Fine structure correction (1) & 1896396.0189 & 2033457.2306 & 8134085.3320 \\
\hline $\begin{array}{l}\text { VP contribution in } 1 \gamma \text { interaction } \\
\text { of order } \alpha(Z \alpha)^{2}\end{array}$ & 1679.2457 & 1959.6265 & 16423.3923 \\
\hline Wichmann-Kroll and LbL correction & -0.0334 & -0.0273 & -0.4388 \\
\hline $\begin{array}{l}\text { 2-loop VP contribution in } 1 \gamma \\
\text { interaction of order } \alpha^{2}(Z \alpha)^{2}\end{array}$ & 12.8421 & 14.9190 & 120.3611 \\
\hline $\begin{array}{l}\text { 3-loop VP contribution in } 1 \gamma \\
\text { interaction of order } \alpha^{3}(Z \alpha)^{2}\end{array}$ & 0.0216 & 0.0263 & 0.2743 \\
\hline $\begin{array}{l}\text { Relativistic corrections taking into } \\
\text { account } 1 \text { - and 2-loop VP in FOPT }\end{array}$ & -0.2018 & -0.2399 & -5.4945 \\
\hline $\begin{array}{l}\text { Relativistic corrections taking into } \\
\text { account } 1 \text { - and 2-loop VP in SOPT }\end{array}$ & 0.6451 & 0.5680 & 18.8617 \\
\hline $\begin{array}{c}\text { 2-loop VP correction in } \\
\text { the SOPT of order } \alpha^{2}(Z \alpha)^{2}\end{array}$ & 1.8811 & 2.3101 & 29.6369 \\
\hline $\begin{array}{l}\text { 3-loop VP correction in the SOPT } \\
\text { and TOPT of order } \alpha^{3}(Z \alpha)^{2}\end{array}$ & 0.0174 & 0.0217 & 0.3317 \\
\hline $\begin{array}{l}\text { Nuclear structure correction } \\
\text { of order }(Z \alpha)^{4}\end{array}$ & $\begin{array}{l}-25.72 \\
\pm 0.04\end{array}$ & $\begin{array}{c}-138.80 \\
\pm 5.79\end{array}$ & $\begin{array}{l}-2774.15 \\
\pm 2.82\end{array}$ \\
\hline $\begin{array}{l}\text { Nuclear structure and 1-loop VP } \\
\text { effects of order } \alpha(Z \alpha)^{4}\end{array}$ & -0.1055 & -0.6014 & -19.1283 \\
\hline $\begin{array}{l}\text { Nuclear structure and 2-loop VP } \\
\text { effects of order } \alpha^{2}(Z \alpha)^{4}\end{array}$ & -0.0042 & -0.0225 & -0.2812 \\
\hline $\begin{array}{c}\text { Nuclear structure effects in } \\
2 \gamma \text { interaction of order }(Z \alpha)^{5}\end{array}$ & 0.1479 & 1.7526 & 78.4300 \\
\hline $\begin{array}{l}\text { Nuclear structure and VP effects in } \\
2 \gamma \text { interaction of order } \alpha(Z \alpha)^{5}\end{array}$ & 0.0030 & 0.0290 & 1.1914 \\
\hline Nucleus polarizability correction & $0.10 \pm 0.02$ & $3.33 \pm 0.10$ & $29.46 \pm 1.20$ \\
\hline $\begin{array}{c}\text { Nuclear structure effects } \\
\text { of order }(Z \alpha)^{6}\end{array}$ & -0.0003 & -0.0014 & -0.1108 \\
\hline $\begin{array}{l}\text { Muon self-energy correction } \\
\text { of order } \alpha(Z \alpha)^{5}\end{array}$ & -4.4953 & -5.4915 & -71.7405 \\
\hline $\begin{array}{l}\text { Muonic 1- and 2-loop VP corrections } \\
\text { in } 1 \gamma \text { interaction and in SOPT }\end{array}$ & 0.1013 & 0.1437 & 2.2850 \\
\hline Recoil correction of order $(Z \alpha)^{5}$ & -0.2843 & -0.1171 & -3.9252 \\
\hline $\begin{array}{c}\text { Radiative and VP corrections } \\
\text { of order }(Z \alpha)^{4}\end{array}$ & -0.0102 & -0.0126 & -0.2016 \\
\hline Hadronic VP correction & 0.0753 & 0.0928 & 1.4853 \\
\hline Summary contribution & 1898060.24 & 2035294.74 & 8147915.57 \\
\hline
\end{tabular}

\section{Conclusion}

In this study, for the first time we have analyzed the fine structure energy interval $(1 S-2 S)$ in light muonic atoms. Various contributions to it of order $\alpha^{3}, \alpha^{4}, \alpha^{5}$ and $\alpha^{6}$ which are important for attaining high accuracy of computation, have been calculated. The analysis of various contributions resulted in the separation of corrections which can be divided into two groups. The first group comprises the contributions specific for each muonic atom, which are asso- 


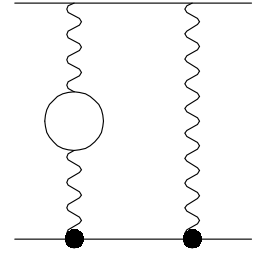

a

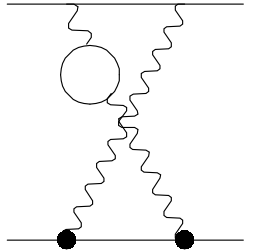

$\mathrm{b}$

Figure 4. Nuclear structure and vacuum polarization correction

ciated with the electronic vacuum polarization and relativistic effects, nuclear structure, and recoil in the first, second, and third orders of the perturbation theory. New contributions to the particle interaction operator of order $\alpha^{5}$ and $\alpha^{6}$ obtained in this study are determined by different effects. The corrections themselves are presented in the integral form for the $S$-states, although the integration with respect to coordinates can always be carried out analytically. Integrations with respect to additional parameters can be carried out either analytically or numerically. The second group includes the integrals known in the analytical form for an arbitrary hydrogen-like atom from the calculations performed primarily for the hydrogen atom. Theoretical uncertainties associated with the values of fundamental physical constants are small and have not been indicated in Tab. 1. The main theoretical errors associated with the nuclear structure and polarizability corrections (see [20]) are given in Tab. 1 explicitly.

The work is supported by Russian Science Foundation (grant No. RSF 18-12-00128).

\section{References}

[1] A. Antognini, F. Kottmann, F. Biraben, et al., Ann. Phys. 331, 127 (2013)

[2] M. Diepold, B. Franke, J. J. Krauth, et al., Ann. Phys. 396, 220 (2018)

[3] R. Pohl, Jour. Phys. Soc. Japan 85, 091003 (2016).

[4] A. E. Dorokhov et al., EPJ Web Conf. 191, 04001 (2018)

[5] M. I. Eides, H. Grotch and V. A. Shelyuto, Phys. Rep. 342, 62 (2001)

[6] A. P. Martynenko, Jour. Exp. Theor. Phys. 101, 1021 (2005)

[7] A. A. Krutov et al., Jour. Exp. Theor. Phys. 120, 73 (2015)

[8] K. Pachucki, Phys. Rev. A 54, 1994 (1996)

[9] E. Borie, Ann. Phys. 327, 733 (2012)

[10] J. L. Friar, G. L. Payne, Phys. Rev. C 72, 014002 (2005)

[11] C. E. Carlson, M. Gorchtein and M. Vanderhaeghen, Phys. Rev A 89, 022504 (2014)

[12] C. Peset and A. Pineda, JHEP 1704, 060 (2017)

[13] R. N. Faustov et al., Phys. Rev. A 90, 012520 (2014)

[14] R. N. Faustov et al., Phys. Lett. B 733, 354 (2014)

[15] A. A. Krutov and A. P. Martynenko, Phys. Rev. A 84, 052514 (2011)

[16] S. G. Karshenboim et al., J. Phys. Chem. Ref. Data 44, 031202 (2015)

[17] S. G. Karshenboim et al., Phys. Rev. A 81, 060501 (2010)

[18] H.F. Hameka, Jour. Chem. Phys. 47, 2728 (1967)

[19] A.P. Martynenko, Phys. Rev. A 76, 012505 (2007)

[20] C. Ji, S. Bacca, N. Barnea, et al., J. Phys. G 45, 093002 (2018)

[21] A. E. Dorokhov et al., Phys. Rev. A 98, 042501 (2018) 